\title{
Data report: biogenic silica deposition in the eastern equatorial Pacific ${ }^{1}$
}

\author{
Oscar E. Romero²
}

\section{Chapter contents}

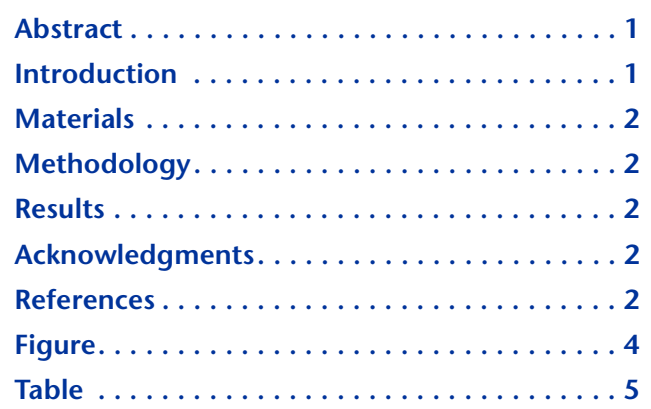

${ }^{1}$ Romero, O.E., 2013. Data report: biogenic silica deposition in the eastern equatorial Pacific. In Pälike, H., Lyle, M., Nishi, H., Raffi, I., Gamage, K., Klaus, A., and the Expedition 320/321 Scientists, Proc. IODP, 320/321: Tokyo (Integrated Ocean Drilling Program Management International, Inc.). doi:10.2204/iodp.proc.320321.206.2013 2Instituto Andaluz de Ciencias de la Tierra (CSICUGR), Avenida de las Palmeras 4, 18100 ArmillaGranada, Spain. oromero@ugr.es

\section{Abstract}

Long-time records of biogenic silica (opal) help researchers to evaluate the effects of global climatic, tectonic, and geochemical Cenozoic events on biological paleoproductivity. Integrated Ocean Drilling Program Expedition 321 Site U1337 contains expanded, nearly continuous records of diatomaceous sediment deposited in deep waters of the eastern equatorial Pacific during the Neogene. In this study, the variability of the biogenic silica at Site U1337 is described by examining temporal changes between the latest middle Miocene and the latest Pleistocene. Periods of highest opal content match well the occurrence of diatom-rich, laminated sediment of the early late Miocene and the late MiocenePliocene transition. Lowest contents are measured in the middle Miocene.

\section{Introduction}

Biogenic silica (opal) produced by diatoms, silicoflagellates, radiolarians, and sponges is a major constituent of marine sediments and an important parameter for geochemical and paleoceanographic studies. Because of the observed links between diatom production and export productivity (Romero and Armand, 2010), biogenic silica is an indicator of the efficiency of the biological pump and export production (Dugdale et al., 1995).

High-resolution records of sedimentary proxies provide insights into fine-scale geochemical and biological responses to climate forcing. In conjunction with shipboard (geophysical/geochemical) measurements, shore-based biogenic silica records allow the high-resolution evaluation of paleoproductivity variations in surface waters and dissolution effects on long timescales. This provides researchers additional data sets that help to evaluate the effect of global climatic, tectonic, and geochemical events that were typical of the Neogene (Cortese et al., 2004) on the regional biological paleoproductivity.

One of the important accomplishments of Integrated Ocean Drilling Program (IODP) Expedition 321 was to recover continuous Neogene sedimentary sections. These represent the only complete Neogene sections in the equatorial Pacific that have high enough sedimentation rates to resolve orbitally forced paleoproductivity cycles (Pälike et al., 2010). To assess changes in (siliceous) paleoproductivity through time, the content of biogenic silica was de- 
termined in sediment from Site U1337. Our primary target for this and future work at this site is understanding the long-term relationship between the sedimentary signature of biogenic silica and climate and between tectonic and biogeochemical signatures during the Neogene in the eastern equatorial Pacific.

\section{Materials}

For this study, we used samples from Holes U1337A $\left(3^{\circ} 50.0065^{\prime} \mathrm{N}, 123^{\circ} 12.3558^{\prime} \mathrm{W}\right.$; water depth $=4472.0$ $\mathrm{m}), \quad \mathrm{U} 1337 \mathrm{~B}\left(3^{\circ} 50.0067^{\prime} \mathrm{N}, 123^{\circ} 12.3621^{\prime} \mathrm{W}\right.$; water depth $=4472.0 \mathrm{~m})$, and U1337C $\left(3^{\circ} 50.0067^{\prime} \mathrm{N}\right.$, $123^{\circ} 12.3755^{\prime} \mathrm{W}$; water depth $\left.=4478.6 \mathrm{~m}\right)$. Samples for the analysis of biogenic silica were freeze-dried and ground in an agate mortar.

\section{Methodology}

All biogenic silica (opal) analyses were conducted at the Center for Marine Environmental Sciences (MARUM; University of Bremen, Bremen, Germany). Opal procedures followed Müller and Schneider (1993), who modified the method originally proposed by DeMaster (1981). The wet-chemical leaching technique involves the extraction of biogenic silica using hot alkaline solutions, measurements by using inductively coupled plasma-emission spectroscopy (ICP-ES) coulometry, and correction for nonbiogenic silica released from coexisting aluminosilicates and quartz (Müller and Schneider, 1993).

An important advantage of the automated extraction method used in our measurements is that the method provides complete biogenic silica recovery and corrects for the nonbiogenic silica. The method determines only one element ( $\mathrm{Si}$ ), and no assumption with respect to the sediment composition has to be made (Müller and Schneider, 1993). A weak point in the manual technique is that the linear slope and the extrapolated intercept values are based on only a few measurements (usually three or four). Based on replicate measurements of silicon standard solutions, the precision of the analytical system is better than $+0.5 \%$ (Müller and Schneider, 1993).

\section{Results}

The results are represented in Figure F1. A total of 364 measurements were conducted on samples from between 4.2 and 258.8 meters below seafloor (mbsf) (Table T1). According to the ship-based stratigraphic model, this interval extends from the latest middle Miocene to the latest Pleistocene (Pälike et al., 2010). The data show relatively large variability: bio- genic silica contents across all samples vary between 1.9 and $69.5 \mathrm{wt} \%$ (average $=16.0 \mathrm{wt} \%)($ Table T1).

Fine-scale variability occurs throughout the studied interval. Although measurements between the uppermost part of the sediment column at Site U1337 downhole to $\sim 260$ mbsf are not completely continuous, some general trends are recognized. Highest contents occur between 228 and 185 mbsf (uppermost interval of the late Miocene) and 108 and 93 mbsf (corresponding to the Miocene-Pliocene transition). Both intervals of highest biogenic silica content match the occurrence of diatom-rich laminations at Site U1337 (Pälike et al., 2010) (Fig. F1). Following the low values between 170 and 165 mbsf, biogenic silica content increases uphole until 110 mbsf. The upper half of the lower Pliocene contains more biogenic silica in than during the lower half, and the range of biogenic silica during the Pleistocene resembles that of the uppermost Pliocene. The lowest biogenic silica contents occur in the uppermost middle Miocene (254-240 mbsf).

Future work on Site U1337 will include filling the gaps (Fig. F1), extending the measurements downhole to the lowermost Neogene, applying an age model, calculating the mass accumulation rate of biogenic silica, and evaluating the spectral evolution. Additionally, we will compare our results to other records from Site U1337, and from high latitudes, in order to establish possible interhemispheric connections.

\section{Acknowledgments}

This research used samples provided by the Integrated Ocean Drilling Program (IODP). Marco Klann (MARUM) performed the analyses. Financial support was provided by the Spanish Scientific Research Council (CGL2007-31136-E/BTE). Reviewer comments from Julia Shackford and an anonymous reviewer are deeply appreciated.

\section{References}

Cortese, G., Gersonde, R., Hillenbrand, C.-D., and Kuhn, G., 2004. Opal sedimentation shifts in the world ocean over the last 15 Myr. Earth Planet. Sci. Lett., 224(34):509-527. doi:10.1016/j.eps1.2004.05.035

DeMaster, D.J., 1981. The supply and accumulation of silica in the marine environment. Geochim. Cosmochim. Acta, 45(10):1715-1732. doi:10.1016/ 0016-7037(81)90006-5

Dugdale, R.C., Wilkerson, F.P., and Minas, H.J., 1995. The role of a silicate pump in driving new production. DeepSea Res., Part 1, 42(5):697-719. doi:10.1016/09670637(95)00015-X 
Müller, P.J., and Schneider, R., 1993. An automated leaching method for the determination of opal in sediments and particulate matter. Deep-Sea Res., 40:425-444.

Pälike, H., Nishi, H., Lyle, M., Raffi, I., Gamage, K., Klaus, A., and the Expedition 320/321 Scientists, 2010. Expedition 320/321 summary. In Pälike, H., Lyle, M., Nishi, H., Raffi, I., Gamage, K., Klaus, A., and the Expedition 320/ 321 Scientists, Proc. IODP, 320/321: Tokyo (Integrated Ocean Drilling Program Management International, Inc.). doi:10.2204/iodp.proc.320321.101.2010
Romero, O.E., and Armand, L.K., 2010. Marine diatoms as indicators of modern changes in oceanographic conditions. In Smol, J.P., and Stoermer, E.F. (Eds.), The Diatoms: Applications for the Environmental and Earth Sciences (2nd ed.): Cambridge (Cambridge Univ. Press), 373-400.

Initial receipt: 2 June 2012

Acceptance: 17 October 2012

Publication: 18 January 2013

MS 320321-206 
Figure F1. Plot of biogenic silica (opal) content vs. depth, Site U1337. Yellow areas define the downhole occurrence of diatom-rich laminated oozes (LDO) (Pälike et al., 2010).

Biogenic silica (wt\%)

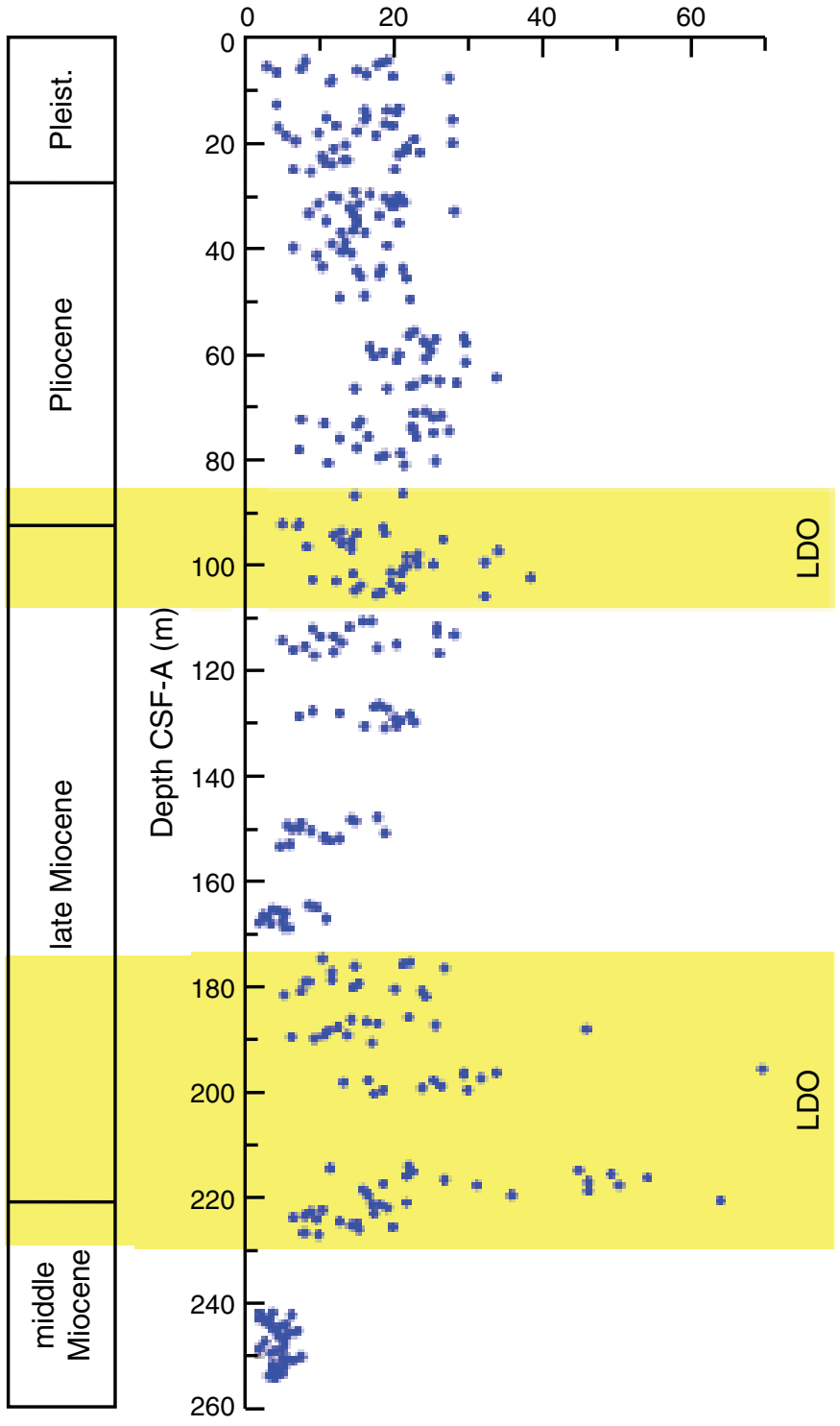


Table T1. Biogenic silica (opal) content, Site U1337. (Continued on next two pages.)

\begin{tabular}{|c|c|c|}
\hline $\begin{array}{l}\text { Hole, core, section, } \\
\text { interval }(\mathrm{cm})\end{array}$ & $\begin{array}{l}\text { Depth } \\
\text { (mbsf) }\end{array}$ & $\begin{array}{l}\text { Biogenic silica } \\
\quad \text { (wt\%) }\end{array}$ \\
\hline \multicolumn{3}{|l|}{$321-$} \\
\hline U1337B-1H-3, 70-71 & 4.16 & 8.14 \\
\hline U1337B-1H-3, 100-101 & 4.46 & 19.06 \\
\hline U1337B-1H-3, 130-131 & 4.76 & 18.37 \\
\hline U1337A-2H-3, 130-31 & 5.05 & 17.92 \\
\hline U1337A-2H-4, 40-41 & 5.55 & 3.06 \\
\hline U1337A-2H-4, 70-71 & 5.85 & 7.54 \\
\hline U1337A2-H-4, 100-101 & 6.15 & 15.06 \\
\hline U1337A-2H-4, 130-131 & 6.45 & 4.33 \\
\hline U1337A-2H-5, 40-41 & 6.95 & 16.32 \\
\hline U1337A-2H-5, 70-71 & 7.25 & 19.95 \\
\hline U1337A-2H-5, 100-101 & 7.55 & 27.28 \\
\hline U1337A-2H-5, 130-131 & 7.85 & 11.77 \\
\hline U1337A-2H-6, 40-41 & 8.35 & 11.47 \\
\hline U1337A-3H-1, 130-131 & 12.65 & 4.32 \\
\hline U1337A-3H-2, 40-41 & 13.25 & 20.74 \\
\hline U1337A-3H-2, 70-71 & 13.55 & 19.13 \\
\hline U1337A-3H-2, 100-101 & 13.85 & 16.11 \\
\hline U1337A-3H-2, 130-131 & 14.15 & 20.35 \\
\hline U1337A-3H-3, 40-41 & 14.75 & 16.28 \\
\hline U1337A-3H-3, 70-71 & 15.05 & 11.00 \\
\hline U1337A-3H-3, 100-101 & 15.35 & 16.09 \\
\hline U1337A-3H-3, 130-131 & 15.65 & 27.94 \\
\hline U1337A-3H-4, 40-41 & 16.15 & 19.04 \\
\hline U1337A-3H-4, 70-71 & 16.45 & 20.03 \\
\hline U1337A-3H-4, 100-101 & 16.75 & 12.26 \\
\hline U1337A-3H-4, 130-131 & 17.05 & 4.63 \\
\hline U1337A-3H-5, 40-41 & 17.65 & 15.14 \\
\hline U1337A-3H-5, 70-71 & 17.95 & 9.98 \\
\hline U1337A-3H-5, 100-101 & 18.25 & 17.65 \\
\hline U1337A-3H-5, 130-131 & 18.55 & 5.46 \\
\hline U1337A-3H-6, 40-41 & 19.15 & 22.66 \\
\hline U1337A-3H-6, 70-71 & 19.45 & 6.85 \\
\hline U1337B-3H-2, 40-41 & 19.99 & 27.84 \\
\hline U1337B-3H-2, 70-71 & 20.29 & 13.51 \\
\hline U1337B-3H-2, 100-101 & 20.59 & 21.78 \\
\hline U1337B-3H-2, 130-131 & 20.89 & 12.06 \\
\hline U1337B-3H-3, 40-41 & 21.49 & 21.65 \\
\hline U1337B-3H-3, 70-71 & 21.79 & 23.60 \\
\hline U1337B-3H-3, 100-101 & 22.09 & 20.82 \\
\hline U1337B-3H-3, 130-131 & 22.39 & 10.34 \\
\hline U1337B-3H-4, 40-41 & 22.99 & 13.16 \\
\hline U1337B-3H-4, 70-71 & 23.29 & 13.70 \\
\hline U1337B-3H-4, 100-101 & 23.59 & 10.77 \\
\hline U1337B-3H-4, 130-131 & 23.89 & 11.71 \\
\hline U1337B-3H-5, 70-71 & 24.79 & 20.19 \\
\hline U1337B-3H-5, 100-101 & 25.09 & 6.56 \\
\hline U1337B-3H-5, 130-131 & 25.39 & 8.92 \\
\hline U1337A-4H-4, 40-41 & 29.40 & 14.78 \\
\hline U1337A-4H-4, 70-71 & 29.70 & 16.89 \\
\hline U1337A-4H-4, 100-101 & 30.00 & 11.73 \\
\hline U1337A-4H-4, 130-131 & 30.30 & 12.47 \\
\hline U1337A-4H-5, 40-41 & 30.90 & 21.46 \\
\hline U1337A-4H-5, 70-71 & 31.20 & 20.08 \\
\hline U1337A-4H-5, 100-101 & 31.50 & 19.81 \\
\hline U1337A-4H-5, 130-131 & 31.80 & 20.12 \\
\hline U1337B-4H-1, 70-71 & 30.10 & 20.65 \\
\hline U1337B-4H-1, 100-101 & 30.40 & 18.94 \\
\hline U1337A-4H-1, 130-131 & 30.70 & 20.12 \\
\hline U1337B-4H-2, 40-41 & 31.30 & 15.44 \\
\hline U1337B-4H-2, 70-71 & 31.60 & 9.99 \\
\hline U1337B-4H-2, 100-101 & 31.90 & 19.64 \\
\hline U1337B-4H-2, 130-131 & 32.20 & 14.00 \\
\hline U1337B-4H-3, 40-41 & 32.80 & 28.11 \\
\hline U1337B-4H-3, 70-71 & 33.10 & 8.79 \\
\hline U1337B-4H-3, 100-101 & 33.40 & 14.54 \\
\hline U1337B-4H-3, 130-131 & 33.70 & 18.28 \\
\hline U1337B-4H-4, 40-41 & 34.30 & 15.08 \\
\hline U1337B-4H-4, 70-71 & 34.60 & 10.92 \\
\hline
\end{tabular}

\begin{tabular}{|c|c|c|}
\hline $\begin{array}{l}\text { Hole, core, section, } \\
\text { interval }(\mathrm{cm})\end{array}$ & $\begin{array}{l}\text { Depth } \\
\text { (mbsf) }\end{array}$ & $\begin{array}{l}\text { Biogenic silica } \\
\quad(w t \%)\end{array}$ \\
\hline U1337B-4H-4, 100-101 & 34.90 & 20.75 \\
\hline U1337B-4H-4, 130-130 & 35.20 & 15.05 \\
\hline U1337B-4H-5, 100-101 & 36.40 & 14.53 \\
\hline U1337B-4H-5, 130-131 & 36.70 & 16.13 \\
\hline U1337A-5H-2, 130-131 & 36.90 & 12.97 \\
\hline U1337B-5H-1, 40-41 & 38.78 & 13.44 \\
\hline U1337B-5H-1, 70-71 & 39.08 & 11.63 \\
\hline U1337B-5H-1, 100-101 & 39.38 & 19.31 \\
\hline U1337B-5H-1, 130-131 & 39.68 & 6.71 \\
\hline U1337B-5H-2, 40-41 & 40.28 & 13.17 \\
\hline U1337B-5H-2, 70-71 & 40.58 & 13.14 \\
\hline U1337B-5H-2, 100-101 & 40.88 & 14.26 \\
\hline U1337B-5H-2, 130-131 & 41.18 & 9.58 \\
\hline U1337B-5H-4, 40-41 & 43.28 & 10.55 \\
\hline U1337B-5H-4, 70-71 & 43.58 & 21.34 \\
\hline U1337B-5H-4, 100-101 & 43.88 & 18.41 \\
\hline U1337B-5H-4, 130-131 & 44.18 & 15.06 \\
\hline U1337B-5H-5, 40-41 & 44.78 & 18.08 \\
\hline U1337B-5H-5, 70-71 & 45.08 & 15.60 \\
\hline U1337B-5H-5, 100-101 & 45.38 & 21.64 \\
\hline U1337A-6H-4, 70-41 & 48.85 & 16.09 \\
\hline U1337A-6H-4, 100-101 & 49.15 & 12.78 \\
\hline U1337A-6H-4, 130-131 & 49.45 & 22.20 \\
\hline U1337A-6H-5, 70-71 & 50.58 & 22.36 \\
\hline U1337B-6H-5, 40-41 & 55.65 & 22.85 \\
\hline U1337A-7H-1, 70-71 & 56.32 & 21.88 \\
\hline U1337A-7H-1, 100-101 & 56.62 & 29.57 \\
\hline U1337A-7H-1, 130-131 & 56.92 & 25.66 \\
\hline U1337A-7H-2, 40-41 & 57.52 & 24.08 \\
\hline U1337A-7H-2, 70-71 & 57.82 & 29.74 \\
\hline U1337A-7H-2, 100-101 & 58.12 & 24.53 \\
\hline U1337A-7H-2, 130-131 & 58.42 & 17.00 \\
\hline U1337A-7H-3, 40-41 & 59.02 & 16.88 \\
\hline U1337A-7H-3, 70-71 & 59.32 & 25.02 \\
\hline U1337A-7H-3, 100-101 & 59.62 & 18.70 \\
\hline U1337A-7H-3, 130-131 & 59.92 & 20.76 \\
\hline U1337A-7H-4, 40-41 & 60.52 & 17.45 \\
\hline U1337A-7H-4, 70-71 & 60.82 & 24.33 \\
\hline U1337A-7H-4, 100-101 & 61.12 & 20.37 \\
\hline U1337A-7H-4, 130-131 & 61.42 & 29.75 \\
\hline U1337B-7H-4, 40-41 & 64.43 & 33.81 \\
\hline U1337B-7H-4, 70-71 & 64.73 & 24.31 \\
\hline U1337B-7H-4, 100-101 & 65.03 & 26.15 \\
\hline U1337B-7H-4, 130-131 & 65.33 & 28.45 \\
\hline U1337B-7H-5, 40-41 & 65.73 & 22.87 \\
\hline U1337B-7H-5, 70-71 & 66.03 & 22.36 \\
\hline U1337B-7H-5, 100-101 & 66.33 & 19.26 \\
\hline U1337B-7H-5, 130-131 & 66.63 & 14.72 \\
\hline U1337A-8H-4, 40-41 & 70.92 & 24.28 \\
\hline U1337A-8H-4, 70-71 & 71.22 & 22.80 \\
\hline U1337A-8H-4, 100-101 & 71.52 & 26.28 \\
\hline U1337A-8H-4, 130-131 & 71.82 & 25.42 \\
\hline U1337B-8H-3, 40-41 & 72.37 & 7.53 \\
\hline U1337B-8H-3, 70-71 & 72.67 & 15.50 \\
\hline U1337B-8H-3, 100-101 & 72.97 & 10.71 \\
\hline U1337B-8H-3, 130-131 & 73.27 & 14.99 \\
\hline U1337B-8H-4, 40-41 & 73.87 & 22.52 \\
\hline U1337B-8H-4, 70-71 & 74.17 & 22.61 \\
\hline U1337B-8H-4, 100-101 & 74.47 & 27.46 \\
\hline U1337B-8H-4, 130-131 & 74.77 & 25.31 \\
\hline U1337B-8H-5, 40-41 & 75.37 & 22.96 \\
\hline U1337B-8H-5, 70-71 & 75.67 & 16.72 \\
\hline U1337B-8H-5, 100-101 & 75.97 & 12.71 \\
\hline U1337A-9H-1, 100-101 & 77.76 & 15.10 \\
\hline U1337A-9H-1, 130-131 & 78.06 & 7.39 \\
\hline U1337A-9H-2, 40-41 & 78.66 & 20.97 \\
\hline U1337A-9H-2, 100-101 & 79.26 & 19.01 \\
\hline U1337A-9H-3, 130-131 & 79.56 & 18.10 \\
\hline U1337A-9H-4, 40-41 & 80.16 & 25.54 \\
\hline
\end{tabular}


Table T1 (continued). (Continued on next page.)

\begin{tabular}{|c|c|c|}
\hline $\begin{array}{l}\text { Hole, core, section, } \\
\text { interval }(\mathrm{cm})\end{array}$ & $\begin{array}{l}\text { Depth } \\
\text { (mbsf) }\end{array}$ & $\begin{array}{c}\text { Biogenic silica } \\
\text { (wt\%) }\end{array}$ \\
\hline U1337A-9H-4, 100-101 & 80.76 & 11.26 \\
\hline U1337A-9H-4, 130-131 & 81.06 & 21.62 \\
\hline U1337В-9H-4, 40-41 & 86.30 & 21.24 \\
\hline U1337В-9H-4, 70-71 & 86.60 & 14.75 \\
\hline U1337A-10H-4, 40-41 & 92.03 & 7.31 \\
\hline U1337A-10H-4, 70-71 & 92.33 & 5.12 \\
\hline U1337A-10H-4, 100-101 & 92.63 & 7.12 \\
\hline U1337A-10H-4, 130-131 & 92.93 & 18.60 \\
\hline U1337A-10H-5, 40-41 & 93.53 & 12.91 \\
\hline U1337A-10H-5, 70-71 & 93.83 & 15.07 \\
\hline U1337A-10H-5, 100-101 & 94.13 & 18.99 \\
\hline U1337A-10H-5, 130-131 & 94.43 & 12.01 \\
\hline U1337B-10H-1, 70-71 & 95.23 & 26.72 \\
\hline U1337B-10H-1, 100-101 & 95.53 & 14.41 \\
\hline U1337B-10H-1, 130-131 & 95.83 & 12.94 \\
\hline U1337B-10H-2, 40-41 & 96.43 & 8.52 \\
\hline U1337B-10H-2, 70-71 & 96.73 & 14.37 \\
\hline U1337B-10H-2, 100-101 & 97.03 & 14.41 \\
\hline U1337B-10H-2, 130-131 & 97.33 & 33.98 \\
\hline U1337B-10H-3, 70-71 & 98.13 & 23.41 \\
\hline U1337B-10H-3, 100-101 & 98.43 & 21.78 \\
\hline U1337B-10H-3, 130-131 & 98.73 & 22.94 \\
\hline U1337B-10H-4, 40-41 & 99.33 & 32.26 \\
\hline U1337B-10H-4, 70-71 & 99.63 & 25.35 \\
\hline U1337A-11H-1, 100-101 & 99.93 & 23.20 \\
\hline U1337A-11H-1, 130-131 & 100.23 & 21.80 \\
\hline U1337A-11H-2, 40-41 & 100.83 & 21.31 \\
\hline U1337A-11H-2, 70-71 & 101.13 & 19.68 \\
\hline U1337A-11H-2, 100-101 & 101.43 & 14.44 \\
\hline U1337A-11H-2, 130-131 & 101.73 & 20.92 \\
\hline U1337A-11H-3, 40-41 & 102.33 & 38.32 \\
\hline U1337A-11H-3, 70-71 & 102.63 & 9.15 \\
\hline U1337A-11H-3, 100-101 & 102.93 & 12.27 \\
\hline U1337A-11H-3, 130-131 & 103.23 & 19.73 \\
\hline U1337A-11H-4, 40-41 & 103.83 & 15.57 \\
\hline U1337A-11H-4, 70-71 & 104.13 & 21.10 \\
\hline U1337A-11H-4, 100-101 & 104.43 & 20.83 \\
\hline U1337A-11H-4, 130-131 & 104.73 & 14.85 \\
\hline U1337A-11H-5, 40-41 & 105.33 & 18.42 \\
\hline U1337A-11H-5, 70-71 & 105.63 & 17.68 \\
\hline U1337A-11H-5, 100-101 & 105.93 & 32.15 \\
\hline U1337A-12H-1, 100-101 & 110.46 & 15.84 \\
\hline U1337A-12H-1, 130-131 & 110.76 & 17.01 \\
\hline U1337A-12H-2, 70-71 & 111.56 & 25.90 \\
\hline U1337A-12H-2, 100-101 & 111.86 & 14.02 \\
\hline U1337A-12H-2, 130-131 & 112.16 & 9.18 \\
\hline U1337A-12H-3, 40-41 & 112.76 & 25.98 \\
\hline U1337A-12H-3, 70-71 & 113.06 & 28.12 \\
\hline U1337A-12H-3, 100-101 & 113.36 & 10.16 \\
\hline U1337A-12H-3, 130-131 & 113.66 & 12.03 \\
\hline U1337A-12H-4, 40-41 & 114.26 & 5.19 \\
\hline U1337A-12H-4, 70-71 & 114.56 & 13.15 \\
\hline U1337A-12H-4, 100-101 & 114.86 & 20.55 \\
\hline U1337A-12H-4, 130-131 & 115.16 & 8.07 \\
\hline U1337A-12H-5, 40-41 & 115.76 & 17.84 \\
\hline U1337A-12H-5, 70-71 & 116.06 & 6.64 \\
\hline U1337A-12H-5, 100-101 & 116.36 & 11.98 \\
\hline U1337A-12H-5, 130-131 & 116.66 & 26.07 \\
\hline U1337A-12H-6, 40-41 & 117.26 & 9.50 \\
\hline U1337B-13H-1, 100-101 & 126.44 & 18.12 \\
\hline U1337B-13H-1, 130-131 & 126.74 & 17.41 \\
\hline U1337B-13H-2, 40-41 & 127.34 & 19.26 \\
\hline U1337B-13H-2, 70-71 & 127.64 & 9.09 \\
\hline U1337B-13H-2, 100-101 & 127.94 & 12.88 \\
\hline U1337B-13H-2, 130-131 & 128.24 & 22.36 \\
\hline U1337B-13H-3, 40-41 & 128.84 & 7.46 \\
\hline U1337B-13H-3, 70-71 & 129.14 & 20.32 \\
\hline U1337B-13H-3, 100-101 & 129.44 & 20.99 \\
\hline U1337B-13H-3, 130-131 & 129.74 & 22.86 \\
\hline
\end{tabular}

\begin{tabular}{|c|c|c|}
\hline $\begin{array}{l}\text { Hole, core, section, } \\
\text { interval }(\mathrm{cm})\end{array}$ & $\begin{array}{l}\text { Depth } \\
\text { (mbsf) }\end{array}$ & $\begin{array}{c}\text { Biogenic silica } \\
\text { (wt\%) }\end{array}$ \\
\hline U1337B-13H-4, 40-41 & 130.34 & 16.08 \\
\hline U1337B-13H-4, 70-71 & 130.64 & 20.46 \\
\hline U1337B-13H-4, 100-101 & 130.94 & 18.94 \\
\hline U1337B-15H-1, 130-131 & 147.75 & 17.82 \\
\hline U1337B-15H-2, 40-41 & 148.35 & 14.43 \\
\hline U1337B-15H-2, 70-71 & 148.65 & 14.80 \\
\hline U1337B-15H-2, 100-101 & 148.95 & 7.66 \\
\hline U1337B-15H-2, 130-131 & 149.25 & 5.86 \\
\hline U1337B-15H-3, 40-41 & 149.85 & 6.43 \\
\hline U1337B-15H-3, 70-71 & 150.15 & 7.48 \\
\hline U1337B-15H-3, 100-101 & 150.45 & 8.92 \\
\hline U1337B-15H-3, 130-131 & 150.75 & 18.80 \\
\hline U1337B-15H-4, 40-41 & 151.35 & 10.82 \\
\hline U1337B-15H-4, 70-71 & 151.65 & 10.80 \\
\hline U1337B-15H-4, 100-101 & 151.95 & 12.82 \\
\hline U1337B-15H-4, 130-131 & 152.25 & 11.61 \\
\hline U1337B-15H-5, 40-41 & 152.85 & 5.97 \\
\hline U1337B-15H-5, 70-71 & 153.15 & 4.71 \\
\hline U1337A-17H-2, 70-71 & 164.43 & 8.65 \\
\hline U1337A-17H-2, 100-101 & 164.73 & 9.75 \\
\hline U1337A-17H-2, 130-131 & 165.03 & 3.68 \\
\hline U1337A-17H-3, 40-41 & 165.63 & 4.57 \\
\hline U1337A-17H-3, 70-71 & 165.93 & 5.49 \\
\hline U1337A-17H-3, 100-101 & 166.23 & 2.38 \\
\hline U1337A-17H-3, 130-131 & 166.53 & 3.05 \\
\hline U1337A-17H-4, 40-41 & 167.13 & 11.04 \\
\hline U1337A-17H-4, 70-71 & 167.43 & 4.97 \\
\hline U1337A-17H-4, 100-101 & 167.73 & 1.99 \\
\hline U1337A-17H-4, 130-131 & 168.03 & 3.62 \\
\hline U1337A-17H-5, 40-51 & 168.63 & 6.07 \\
\hline U1337A-17H-5, 70-71 & 168.93 & 5.24 \\
\hline U1337A-18H-1, 100-101 & 174.56 & 10.41 \\
\hline U1337A-18H-2, 40-41 & 175.46 & 22.37 \\
\hline U1337A-18H-2, 70-71 & 175.76 & 21.13 \\
\hline U1337A-18H-2, 100-101 & 176.06 & 14.90 \\
\hline U1337A-18H-2, 130-131 & 176.36 & 26.86 \\
\hline U1337A-18H-3, 40-41 & 176.96 & 11.68 \\
\hline U1337A-18H-4, 40-41 & 178.46 & 11.62 \\
\hline U1337A-18H-4, 70-71 & 178.76 & 8.07 \\
\hline U1337A-18H-4, 100-101 & 179.06 & 8.62 \\
\hline U1337A-18H-4, 130-131 & 179.36 & 15.33 \\
\hline U1337A-18H-5, 40-41 & 179.96 & 14.57 \\
\hline U1337A-18H-5, 70-71 & 180.26 & 20.14 \\
\hline U1337A-18H-5, 100-101 & 180.56 & 7.67 \\
\hline U1337A-18H-5, 130-131 & 180.86 & 23.92 \\
\hline U1337A-18H-6, 40-41 & 181.46 & 5.38 \\
\hline U1337A-18H-6, 70-71 & 181.76 & 24.26 \\
\hline U1337A-19H-2, 40-41 & 185.90 & 21.89 \\
\hline U1337A-19H-2, 70-71 & 186.20 & 14.39 \\
\hline U1337A-19H-2, 100-101 & 186.50 & 16.24 \\
\hline U1337A-19H-2, 130-131 & 186.80 & 17.99 \\
\hline U1337A-19H-3, 40-41 & 187.40 & 25.67 \\
\hline U1337A-19H-3, 70-71 & 187.70 & 12.51 \\
\hline U1337A-19H-3, 100-101 & 188.00 & 45.90 \\
\hline U1337A-19H-3, 130-131 & 188.30 & 11.27 \\
\hline U1337A-19H-4, 40-41 & 188.90 & 13.70 \\
\hline U1337A-19H-4, 70-71 & 189.20 & 10.80 \\
\hline U1337A-19H-4, 100-101 & 189.50 & 6.37 \\
\hline U1337A-19H-4, 130-131 & 189.80 & 9.45 \\
\hline U1337A-19H-5, 40-41 & 190.40 & 17.01 \\
\hline U1337A-20H-1, 130-131 & 195.50 & 69.48 \\
\hline U1337A-20H-2, 40-41 & 196.10 & 33.84 \\
\hline U1337A-20H-2, 70-71 & 196.40 & 29.49 \\
\hline U1337A-20H-2, 100-101 & 196.70 & 29.42 \\
\hline U1337A-20H-3, 40-41 & 197.30 & 31.64 \\
\hline U1337A-20H-3, 70-71 & 197.60 & 25.45 \\
\hline U1337A-20H-3, 100-101 & 197.90 & 16.67 \\
\hline U1337A-20H-3, 130-131 & 198.20 & 13.35 \\
\hline U1337A-20H-4, 40-41 & 198.80 & 26.47 \\
\hline
\end{tabular}


Table T1 (continued).

\begin{tabular}{lcc}
\hline \multicolumn{1}{c}{$\begin{array}{c}\text { Hole, core, section, } \\
\text { interval (cm) }\end{array}$} & $\begin{array}{c}\text { Depth } \\
\text { (mbsf) }\end{array}$ & $\begin{array}{c}\text { Biogenic silica } \\
\text { (wt\%) }\end{array}$ \\
\hline U1337A-20H-4, 70-71 & 199.10 & 23.78 \\
U1337A-20H-4, 100-101 & 199.40 & 29.84 \\
U1337A-20H-4, 130-131 & 199.70 & 18.73 \\
U1337A-20H-5, 40-41 & 200.30 & 17.29 \\
U1337C-6H-1, 70-71 & 213.95 & 21.95 \\
U1337C-6H-1, 100-101 & 214.25 & 11.39 \\
U1337C-6H-1, 130-131 & 214.55 & 44.77 \\
U1337C-6H-2, 40-41 & 215.15 & 22.50 \\
U1337C-6H-2, 70-71 & 215.45 & 49.26 \\
U1337C-6H-2, 100-101 & 215.75 & 21.68 \\
U1337C-6H-2, 130-131 & 216.05 & 53.98 \\
U1337C-6H-3, 40-41 & 216.65 & 26.80 \\
U1337C-6H-3, 70-71 & 216.95 & 46.13 \\
U1337C-6H-3, 10-101 & 217.25 & 18.63 \\
U1337D-22H-1, 40-41 & 217.45 & 50.11 \\
U1337D-22H-1, 70-71 & 217.75 & 31.19 \\
U1337D-22H-1, 130-131 & 218.35 & 15.73 \\
U1337D-22H-2, 40-41 & 218.85 & 46.05 \\
U1337C-7H-1, 70-71 & 219.29 & 35.98 \\
U1337C-7H-1, 100-101 & 219.59 & 16.58 \\
U1337C-7H-1, 130-131 & 219.89 & 52.90 \\
U1337C-7H-2, 40-41 & 220.49 & 63.77 \\
U1337C-7H-2, 70-71 & 220.79 & 21.68 \\
U1337C-7H-2, 100-101 & 221.09 & 17.24 \\
U1337C-7H-2, 130-131 & 221.39 & 18.26 \\
U1337C-7H-3, 40-41 & 221.99 & 19.27 \\
U1337C-7H-3, 70-71 & 222.29 & 10.52 \\
U1337C-7H-3, 100-101 & 222.59 & 8.92 \\
U1337C-7H-3, 130-131 & 222.89 & 17.42 \\
U1337C-7H-4, 40-41 & 223.49 & 8.20 \\
U1337C-7H-4, 70-71 & 223.79 & 6.70 \\
U1337C-7H-4, 100-101 & 224.09 & 9.58 \\
U1337C-7H-4, 130-131 & 224.39 & 12.69 \\
U1337C-7H-5, 40-41 & 224.99 & 15.04 \\
U1337C-7H-5, 70-71 & 225.29 & 14.24 \\
U1337C-7H-5, 100-101 & 225.59 & 20.03 \\
U1337C-7H-5, 130-131 & 225.89 & 15.38 \\
U1337C-7H-6, 40-41 & 226.49 & 8.02 \\
U1337C-7H-6, 70-71 & 226.79 & 8.17 \\
U1337C-7H-6, 100-101 & 227.09 & 9.88 \\
U1337C-9H-2, 40-41 & 241.46 & 2.02 \\
U1337C-9H-2, 70-71 & 241.76 & 3.91 \\
U1337C-9H-2, 100-101 & 242.06 & 6.37 \\
U1337C-9H-2, 130-131 & 242.36 & 2.16 \\
U1337C-9H-3, 40-41 & 242.96 & 2.01 \\
& & \\
& &
\end{tabular}

\begin{tabular}{lcc}
\hline $\begin{array}{c}\text { Hole, core, section, } \\
\text { interval (cm) }\end{array}$ & $\begin{array}{c}\text { Depth } \\
\text { (mbsf) }\end{array}$ & $\begin{array}{c}\text { Biogenic silica } \\
\text { (wt\%) }\end{array}$ \\
\hline U1337C-9H-3, 70-71 & 243.26 & 3.34 \\
U1337C-9H-3, 100-101 & 243.56 & 3.13 \\
U1337C-9H-3, 130-131 & 243.86 & 5.70 \\
U1337C-9H-4, 40-41 & 244.46 & 4.79 \\
U1337C-9H-4, 70-71 & 244.76 & 3.89 \\
U1337C-9H-4, 100-101 & 245.06 & 7.17 \\
U1337C-9H-4, 130-131 & 245.36 & 6.85 \\
U1337C-9H-5, 40-41 & 245.96 & 5.81 \\
U1337C-9H-5, 70-71 & 246.26 & 4.45 \\
U1337A-25X-2, 40-41 & 247.06 & 5.29 \\
U1337A-25X-2, 70-71 & 247.36 & 2.74 \\
U1337A-25X-2, 100-101 & 247.66 & 5.24 \\
U1337A-25X-2, 130-131 & 247.96 & 5.25 \\
U1337A-25X-3, 40-41 & 248.56 & 1.88 \\
U1337A-25X-3, 70-71 & 248.86 & 5.17 \\
U1337A-25X-3, 100-101 & 249.16 & 3.97 \\
U1337A-25X-3, 130-131 & 249.46 & 3.45 \\
U1337A-25X-4, 40-41 & 250.06 & 7.55 \\
U1337A-25X-4, 70-71 & 250.36 & 4.95 \\
U1337A-25X-4, 100-101 & 250.66 & 5.79 \\
U1337A-25X-4, 130-131 & 250.96 & 6.54 \\
U1337B-25H-2, 40-41 & 251.56 & 3.86 \\
U1337B-25H-2, 70-71 & 251.86 & 5.29 \\
U1337B-25H-2, 100-101 & 252.16 & 3.71 \\
U1337B-25H-2, 130-131 & 252.46 & 4.72 \\
U1337B-25H-3, 40-41 & 253.06 & 4.47 \\
U1337B-25H-3, 70-71 & 253.36 & 5.06 \\
U1337B-25H-3, 100-101 & 253.66 & 3.27 \\
U1337B-25H-3, 130-131 & 253.96 & 4.13 \\
U1337B-25H-4, 40-41 & 254.56 & 6.27 \\
U1337B-25H-4, 70-71 & 254.86 & 4.13 \\
U1337B-25H-4, 100-101 & 255.16 & 3.07 \\
U1337B-25H-4, 130-131 & 255.46 & 3.60 \\
U1337A-26X-1, 40-41 & 254.87 & 4.65 \\
U1337A-26X-1, 70-71 & 255.17 & 4.95 \\
U1337A-26X-1, 100-101 & 255.47 & 6.62 \\
U1337A-26X-2, 40-41 & 256.37 & 4.12 \\
U1337A-26X-2, 70-71 & 256.67 & 4.65 \\
U1337A-26X-2, 100-101 & 256.97 & 6.76 \\
U1337A-26X-2, 130-131 & 257.27 & 5.46 \\
U1337A-26X-3, 40-41 & 257.87 & 4.17 \\
U1337A-26X-3, 70-71 & 258.17 & 3.18 \\
U1337A-26X-3, 100-101 & 258.47 & 11.83 \\
U1337A-26X-3, 130-131 & 258.77 & 4.74 \\
\hline & & \\
\hline
\end{tabular}

